\title{
Insight into the ERVK Integrase - Propensity for DNA Damage
}

\author{
Samantha Bray ${ }^{1 \dagger}$, Matthew Turnbull1t, Sherry Hebert ${ }^{1}$ and Renée N. Douville ${ }^{1,2 *}$ \\ ${ }^{1}$ Douville Lab, Department of Biology, University of Winnipeg, Winnipeg, MB, Canada, ${ }^{2}$ Department of Immunology, \\ University of Manitoba, Winnipeg, MB, Canada
}

Retroviruses create permanently integrated proviruses that exist in the host genome. Retroviral genomes encode for functionally conserved gag, pro, pol, and env regions, as well as integrase $(\mathrm{IN})$, which is required for retroviral integration. IN mediates viral genome insertion through $3^{\prime}$ end processing of the viral DNA and the strand transfer reaction. This process requires the formation of a pre-integration complex, comprised of IN, viral DNA, and cellular proteins. Viral insertion causes DNA damage, leading to the requirement of host DNA repair mechanisms. Therefore, a failure of DNA repair pathways may result in genomic instability and potentially cause host cell death. Considering the numerous human diseases associated with genomic instability, the endogenous retrovirus-K (ERVK) IN should be considered as a putative contributor to DNA damage in human cells. Future research and drug discovery should focus on ERVK IN activity and its role in human conditions, such as neurological disease and cancers.

Keywords: endogenous retrovirus-K, integrase, DNA damage, genomic instability, neurological disease, cancer

OPEN ACCESS

Edited by:

Akio Adachi,

Tokushima University, Japan

Reviewed by:

Takao Masuda,

Tokyo Medical and Dental University,

Japan

Yoshinao Kubo,

Nagasaki University, Japan

*Correspondence:

Renée N. Douville r.douville@uwinnipeg.ca

${ }^{+}$Co-first authors

Received: 20 October 2016 Accepted: 18 November 2016 Published: 01 December 2016

Citation:

Bray S, Turnbull M, Hebert S and Douville RN (2016) Insight into the ERVK Integrase - Propensity

for DNA Damage.

Front. Microbiol. 7:1941. doi: 10.3389/fmicb.2016.01941

\section{INTRODUCTION}

Retroviruses have mastered the art of horizontal gene transfer. A key viral enzyme in this process is the retroviral integrase (IN) enzyme which catalyzes the merger of viral and host genomes. Starting from an RNA genome, retroviruses convert their genetic material into double stranded DNA (dsDNA) using a virally encoded reverse transcriptase (RT) enzyme. The viral dsDNA is then transported into the nucleus as part of the pre-integration complex (PIC), which is composed of both viral and host proteins, including IN (reviewed in Jayappa et al., 2012; Gerard et al., 2013). IN coordinates processing of the linear viral DNA ends and joining those ends into target host DNA through a strand transfer reaction (reviewed in Lesbats et al., 2016). Subsequently, DNA lesions are left in the host genome, which require cellular repair mechanisms to restore genomic integrity. Aberrant IN activity or imperfect repair mechanisms can leave a host vulnerable to genomic instability through the accumulation of DNA lesions. This paper provides a perspective on how the endogenous retrovirus-K (ERVK) IN enzyme may play a role in generating genomic instability in the context of human disease.

\section{Structure of Retroviral Integrases}

The structure of a retroviral IN commonly contains three domains which are the N-terminal domain (NTD), the central catalytic domain (CCD), and the C-terminal domain (CTD); some also encode an additional N-terminal extension domain (NED) (reviewed in Lesbats et al., 2016). The NTD is involved in IN multimerization and contains two conserved histidine $(\mathrm{H})$ and cysteine (C) residues that form a highly conserved zinc-binding HHCC motif found in all retroviral IN (Figure 1) (reviewed in Zheng et al., 1996; Lesbats et al., 2016). The CCD contains two highly 


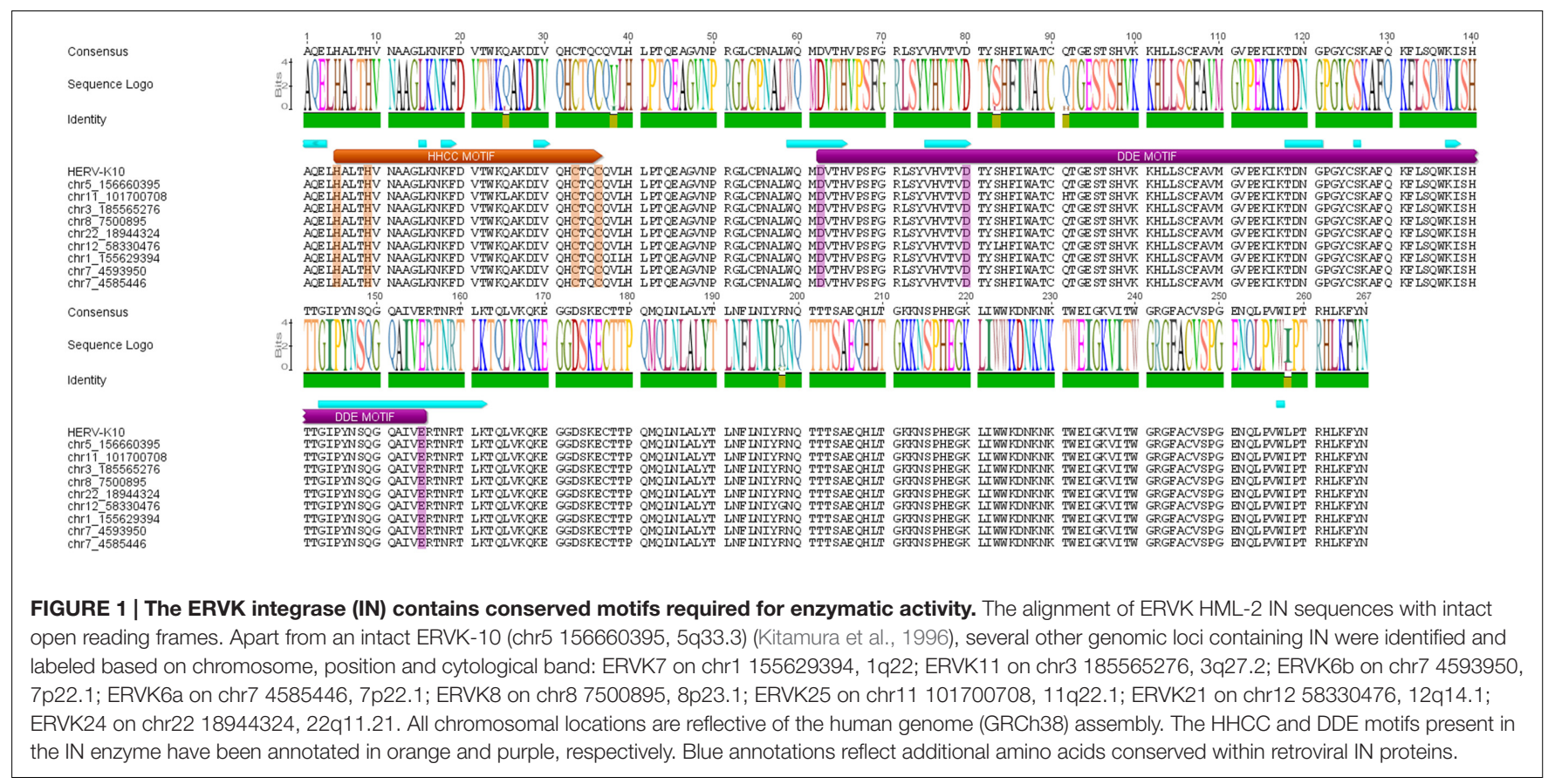

conserved aspartic acid (D) residues and a glutamic acid (E) residue that form a catalytic triad called the DDE motif (Hare et al., 2010). This catalytic triad recognizes and binds to $\mathrm{Mg}^{2+}$ which is essential for proper IN function and multimerization (reviewed in Lesbats et al., 2016). The acidic residues of the DDE motif catalyze $3^{\prime}$ linear DNA processing, DNA strand transfer reactions, and disintegration reactions (reverse of the strand transfer ligation reaction) - all enzymatic processes required for a functional IN (Hossain et al., 2013; Wolkowicz et al., 2014). The CTD is the least conserved domain, although it contains some conserved tryptophan residues, and is integral to the formation of the intasome (reviewed in Lesbats et al., 2016). Post-translational modification of IN enzymes can also impact their activity. For HIV, acetylation of lysine residues within the CTD by cellular proteins, p300 and GCN5, enhances the DNA binding capacity and strand-transfer activity of IN (Cereseto et al., 2005; Suzuki and Chew, 2012). Additionally, integrase enzymes function in higher-order multimeric complexes, as determined by protein analysis and crystallography (Diamond and Bushman, 2005; Li et al., 2011).

Integrase carries out important enzymatic functions that integrate reverse-transcribed viral DNA into host cell DNA. However, IN does not function alone, as the functional PIC also contains host proteins. In HIV and Moloney murine leukemia virus (MoMLV), IN is associated with barrier-toautointegration factor (BAF), which helps prevent integration of the viral DNA into itself (Van Maele et al., 2006; Wiebe and Jamin, 2016). It would be of great interest to determine whether BAF is a universally utilized mechanism by which retroviruses are protected from autointegration, or whether divergent mechanisms have evolved. Retroviruses also utilize host cell proteins in the identification of suitable integration sites (reviewed in Kvaratskhelia et al., 2014). Lens epithelium-derived growth factor (LEDGF) directs HIV IN to target integration sequences and protects IN from proteolysis (Gerard et al., 2013). More recently, the bromo- and extra-terminal domain (BET) proteins have been demonstrated to function in proviral targeting in MoMLV (De Rijck et al., 2013). The varied preferences exhibited for retroviral insertion suggests that ERV targeting will also utilize unique cellular proteins.

\section{Integrase Function and the Consequences of Failed DNA Lesion Repair}

Integration is carried out in three steps: (i) processing, (ii) joining, and (iii) host-mediated DNA repair (reviewed in Lesbats et al., 2016). In the first step of retroviral DNA integration, IN binds to the linear viral dsDNA to form a stable complex called an intasome. IN then cleaves two (or three) nucleotides from the $3^{\prime}$ ends of viral dsDNA to produce $3^{\prime}$ hydroxyl groups. Next, IN coordinates the cleavage of host DNA phosphodiester bonds and their ligation to the free viral hydroxyl groups. Retroviral integration is not a perfect process. The strand transfer complex (STC) leaves behind single-stranded DNA (ssDNA) gaps at the host-provirus junctures and two $5^{\prime}$ base pair overhang extensions of the viral DNA. Cellular DNA repair mechanisms are then required to restore genome integrity (Yoder and Bushman, 2000; reviewed in Lesbats et al., 2016). However, the process by which the STC is disassembled to allow for DNA repair remains unknown.

Accumulation of DNA lesions and genomic instability lead to loss of cellular functionality, and ultimately the death or transformation of human cells (Hanahan and Weinberg, 2011). Unrepaired IN-mediated ssDNA lesions are hotspots for the formation of double stranded breaks (DSB) in the DNA of 
replicating host cells (Ryan et al., 2016). Products of IN activity such as (i) newly integrated proviruses, (ii) viral episomes resulting from autointegration (2LTR loops), and (iii) unresolved lesions in the host genome may signal the host cell to initiate an innate immune response and definitely signal engagement of diverse DNA damage repair pathways critical to successful integration; failure to repair the ssDNA lesions left by IN can induce apoptosis (Stetson et al., 2008; Bregnard et al., 2014; Ryan et al., 2016).

Interestingly, DSBs can enhance the integration of HIV DNA into the host genome when in the presence of catalytically inactive IN (Ebina et al., 2012; Koyama et al., 2013). Complementary findings that IN is dispensable for provirus insertion in the context of DSBs stem from the observation of enhanced integration of wild-type IN virus when DNA-damaged cells were treated with the IN inhibitor raltegravir (Koyama et al., 2013). This study suggests that IN inhibitors are best suited to prevent DNA damage in healthy cells, and that both inhibition of RT (formation of viral DNA) and IN would be required in cells containing DNA lesions.

\section{Mobile Elements in the Human Genome as a Source of Genomic Instability}

The human genome contains many endogenous retroviruses which potentially encode IN (Seifarth et al., 2005). Additionally, enzymes encoded by non-LTR retrotransposons are known to mediate DNA damage in humans (e.g., ORF2p) (Gasior et al., 2006). Select elements, such as ERVK and long interspersed nuclear element-1 (LINE-1) are of special interest because of their retrotranspositional activity in modern humans (Mills et al., 2007). For example, both ERVs and LINE-1 are active in the developing human brain, and then repressed in mature tissues (reviewed in Erwin et al., 2014; Mortelmans et al., 2016). Both ERVK and LINE-1 are negatively regulated by members of the APOBEC3 family (Lee et al., 2008; Jones et al., 2013), and differential activity of these elements has been independently linked to human disease (Frank et al., 2005; Douville et al., 2011; Balestrieri et al., 2014; Bundo et al., 2014). Therefore, it is possible that these mobile elements generate similar patterns of genomic instability when active. Although this paper calls attention to the underappreciated role of ERVK IN, it is not the only endogenous enzyme which may induce DNA damage and subsequent genomic instability.

\section{ERVK Encodes a Functional Integrase}

The original description of a functional IN in ERVK-10 (HERVK10) was by Kitamura et al. (1996). This retroviral enzyme shows not only terminal cleavage and strand transfer activities of ERVK LTR substrates, but also of LTRs from the divergent retroviruses, HIV and RSV (Kitamura et al., 1996). Despite these findings, there has been an underwhelming interest in ERVK IN and their potential role in human biology.

Recently, our team sought to determine if additional ERVK loci related to ERVK-10 (those in the HML-2 clade) encode potentially active IN enzymes. IN sequences were identified in the human genome GRCh38 by a tBLASTn search based on the region of the reconstituted infectious ERVK virus Pheonix (Dewannieux et al., 2006) which matches the Pfam entries for each IN domain: NTD (Integrase_Zn, PF02022), CCD (rve, PF00665), CTD (IN_DBD_C, PF00552). BLAST hits from the same ERV were merged and extended according to their alignment by MACSE. Out of the 20 ERVK proviruses containing a full-length IN ORF, only nine maintained intact HHCC and DDE active site motifs (Figure 1). All putative ERVK IN-encoding ORFs contained signature HALTH (HXXXH) and CTQC (CXXC) sequences for the HHCC motif. Additionally, all but one of these sequences included a WQMD signature associated with the first aspartate, and a TDNG signature was consistently associated with the second aspartate within the $\mathrm{D}\left(\mathrm{X}_{17}\right) \mathrm{D}\left(\mathrm{X}_{35}\right)$ E motif.

Select mutations within the HIV IN have been shown to modulate its catalytic activity (class I mutants) (Engelman, 1999). When comparing amino acid substitutions within mutant HIV IN enzymes and the consensus ERVK IN sequence, we found that ERVK IN contains no known inactivating substitutions that alter the active sites or the activity of $3^{\prime}$ processing, DNA binding or joining (Konsavage et al., 2007; Li et al., 2012; Johnson et al., 2013). However, without a clear understanding of ERVK IN and cellular protein interaction, it is difficult to interpret which substitutions would impact PIC formation and STC disassembly. Further, ERVK IN likely plays a role in processes other than integration, such as enhancement of reverse transcription and virion assembly (reviewed in Lesbats et al., 2016).

A model of a single ERVK IN subunit superimposed on the Mouse Mammary Tumor Virus (MMTV) intasome (Figure 2) shows that the predicted protein folding results in the expected domain architecture for betaretroviral IN (Ballandras-Colas et al., 2016). Moreover, the orientation of key residues forms a clear active site conformation. The DNA binding affinity of the ERVK IN may differ from MMTV IN because of a linker between the NTD and CCD domains that impinges into the DNA binding site, and may partially explain the more relaxed substrate specificity of the ERVK IN toward retroviral LTRs (Kitamura et al., 1996). Without a validated ERVK intasome model, it will be difficult to predict mutations that may impact multimerization and drug resistance (reviewed in Lesbats et al., 2016).

\section{ERVK-Associated Diseases Are Associated with Genomic Instability}

The expression of ERVK IN in human cells is poorly documented. However, cortical brain tissue from patients with amyotrophic lateral sclerosis (ALS) contain an enhanced frequency of ERVK transcripts from proviral loci containing open reading frames (ORFs) for IN, when compared to controls with systemic disease (Douville et al., 2011). Moreover, the presence of functional RT expression in several disease states, including ALS (Douville et al., 2011) and breast cancer (Golan et al., 2008), alludes to the possibility of IN co-expression derived from the cleavage of the ERVK gag-pol polyprotein. The prime limiting factor for the 


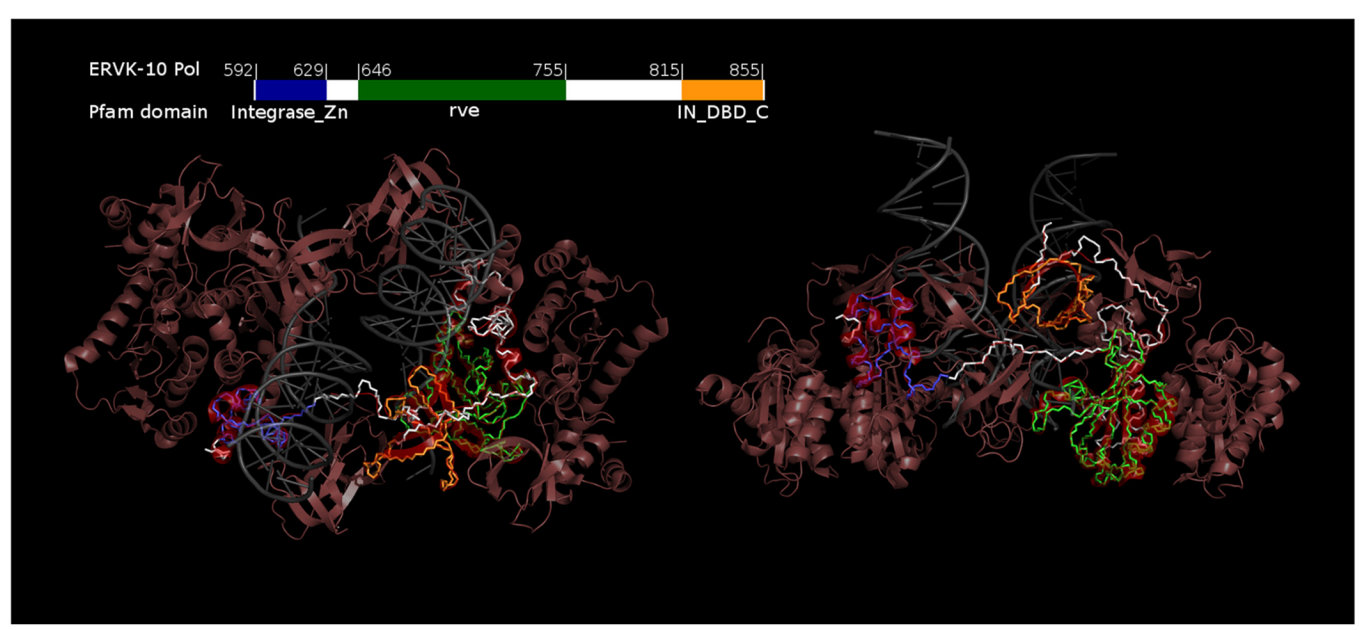

FIGURE 2 | Homology model of the ERVK-10 integrase. The ERVK-10 IN (Uniprot P10266) was modeled by homology to the betaretrovirus Mouse Mammary Tumor Virus (MMTV) intasome (PDB 3JCA). 100 models were produced using Modeller 9.15 using default settings except the maximum distance for inter-alpha-carbon homology derived constraints was 32 Angstroms and the slow default was used for molecular dynamics. The model with the lowest DOPE score (-26429) was superimposed onto the template chain (3JCA:A) by their alpha carbon co-ordinates in pymol, and the RMSD between all 260 aligned alpha carbons is $0.275 \AA$. ERVK backbone carbons and nitrogens are colored so regions matching the Pfam entries Integrase_Zn, rve, and IN_DBD_C match the color of their linear representation on the ERVK-10 sequence. 3JCA is light red except the template chain is bright red, and the DNA is gray. This figure was produced using Pymol and GIMP.

identification of ERVK IN in human tissue samples is the lack of commercially available antibodies for the detection of this viral protein.

Interestingly, genomic instability is a hallmark of several ERVK-associated human diseases, including ALS (Deng et al., 2014; Maizels, 2015), schizophrenia (Smith et al., 2010; Kushima et al., 2016) and cancers (Romanish et al., 2010; Mullins and Linnebacher, 2012). Although proposed mechanisms for DNA damage do exist for these conditions, they do not preclude the involvement of ERVK IN. Conceivably, if the ERVK IN is shown to mediate DNA damage in human disease, this pathology could potentially be averted through the use of viral IN inhibitors (Gunthard et al., 2016; Kanters et al., 2016). Concrete evidence of efficacy against ERVK-driven genomic instability would be a landmark step toward developing viable therapeutic options for the treatment of these diseases.

Moreover, certain genetic backgrounds may be more susceptible to ERVK IN-driven DNA damage. STC disassembly is required for DNA damage response proteins to access lesion sites (reviewed in Lesbats et al., 2016). However, while little is known about the disassembly process, there is reason to believe that this process utilizes host cell proteins to target IN for degradation through ubiquitination or phosphorylation (Mousnier et al., 2007; Suzuki and Chew, 2012). Many of the ERVK-associated diseases are characterized by impairment of ubiquitination or abrogated proteasome function, such as in schizophrenia (Rubio et al., 2013) and ALS (Chang and Monteiro, 2015; Ciechanover and Kwon, 2015). Blockade of these pathways would be consistent with a failure to degrade IN complexes and disallow DNA repair proteins from restoring lesioned areas of the genome. Similarly, direct alterations within DNA repair systems, as seen in numerous forms of cancer (Lord and Ashworth, 2012; O'Connor, 2015), may favor the buildup of DNA damage in the context of ERVK IN expression.

\section{Therapeutic Value of Integrase Inhibitors in ERVK-Associated Disease}

It is tantalizing to imagine how currently used anti-retroviral drugs could be repurposed for the treatment of clinically challenging conditions, such as ALS and cancers. In recent years, the adoption of IN strand transfer inhibitor (InSTI) for the treatment of HIV has come to the forefront (Gunthard et al., 2016), in part due to their improved blood-brain barrier permeability as compared to protease and RT inhibitors (Varatharajan and Thomas, 2009; Meeker et al., 2014; Nightingale et al., 2014). For example, the IN inhibitor dolutegravir has been shown to cross the blood-brain barrier, resulting in therapeutic concentrations in the cerebrospinal fluid (CSF) similar to those found in plasma (Letendre et al., 2014). This highlights the potential use of IN inhibitors for arresting progression of ERVK-associated neurological conditions.

Concern surrounding the rapid appearance of drug-resistance mutations in exogenous retroviruses commonly limits the therapeutic potential of anti-retroviral compounds (Gunthard et al., 2016; Kanters et al., 2016). However, ERVK is a prisoner entrapped by the high fidelity of human genomic replication, thus limiting the appearance of drug resistance mutations and improving the therapeutic potential for IN inhibitors in ERVK-mediated disease. Future identification of candidate IN inhibitors for clinical drug trials in ERVK-associated disease will provide a substantive contribution toward the development 
of effective (and desperately needed) treatment options for refractory conditions, such ALS and cancers.

\section{AUTHOR CONTRIBUTIONS}

MT curated the ERVK IN sequences from the GRCh38 assembly. SB performed ERVK IN alignment. MT produced the ERVK IN protein model. RD conceived the study. SB, MT, SH and RD wrote the manuscript. All authors read and approved the final manuscript.

\section{REFERENCES}

Balestrieri, E., Pitzianti, M., Matteucci, C., D’Agati, E., Sorrentino, R., Baratta, A., et al. (2014). Human endogenous retroviruses and ADHD. World J. Biol. Psychiatry 15, 499-504. doi: 10.3109/15622975.2013.862345

Ballandras-Colas, A., Brown, M., Cook, N. J., Dewdney, T. G., Demeler, B., Cherepanov, P., et al. (2016). Cryo-EM reveals a novel octameric integrase structure for betaretroviral intasome function. Nature 530, 358-361. doi: 10. 1038/nature16955

Bregnard, C., Benkirane, M., and Laguette, N. (2014). DNA damage repair machinery and HIV escape from innate immune sensing. Front. Microbiol. 5:176. doi: 10.3389/fmicb.2014.00176

Bundo, M., Toyoshima, M., Okada, Y., Akamatsu, W., Ueda, J., NemotoMiyauchi, T., et al. (2014). Increased 11 retrotransposition in the neuronal genome in schizophrenia. Neuron 81, 306-313. doi: 10.1016/j.neuron.2013. 10.053

Cereseto, A., Manganaro, L., Gutierrez, M. I., Terreni, M., Fittipaldi, A., Lusic, M., et al. (2005). Acetylation of HIV-1 integrase by p300 regulates viral integration. EMBO J. 24, 3070-3081. doi: 10.1038/sj.emboj.7600770

Chang, L., and Monteiro, M. J. (2015). Defective proteasome delivery of polyubiquitinated proteins by Ubiquilin-2 proteins containing ALS mutations. PLoS ONE 10:e0130162. doi: 10.1371/journal.pone.0130162

Ciechanover, A., and Kwon, Y. T. (2015). Degradation of misfolded proteins in neurodegenerative diseases: therapeutic targets and strategies. Exp. Mol. Med. 47:e147. doi: 10.1038/emm.2014.117

De Rijck, J., de Kogel, C., Demeulemeester, J., Vets, S., El Ashkar, S., Malani, N., et al. (2013). The BET family of proteins targets moloney murine leukemia virus integration near transcription start sites. Cell Rep. 5, 886-894. doi: 10.1016/j. celrep.2013.09.040

Deng, Q., Holler, C. J., Taylor, G., Hudson, K. F., Watkins, W., Gearing, M., et al. (2014). FUS is phosphorylated by DNA-PK and accumulates in the cytoplasm after DNA damage. J. Neurosci. 34, 7802-7813. doi: 10.1523/JNEUROSCI.017214.2014

Dewannieux, M., Harper, F., Richaud, A., Letzelter, C., Ribet, D., Pierron, G., et al. (2006). Identification of an infectious progenitor for the multiple-copy HERV-K human endogenous retroelements. Genome Res. 16, 1548-1556. doi: 10.1101/gr.5565706

Diamond, T. L., and Bushman, F. D. (2005). Division of labor within human immunodeficiency virus integrase complexes: determinants of catalysis and target DNA capture. J. Virol. 79, 15376-15387. doi: 10.1128/JVI.79.24.1537615387.2005

Douville, R., Liu, J., Rothstein, J., and Nath, A. (2011). Identification of active loci of a human endogenous retrovirus in neurons of patients with amyotrophic lateral sclerosis. Ann. Neurol. 69, 141-151. doi: 10.1002/ana.22149

Ebina, H., Kanemura, Y., Suzuki, Y., Urata, K., Misawa, N., and Koyanagi, Y. (2012). Integrase-independent HIV-1 infection is augmented under conditions of DNA damage and produces a viral reservoir. Virology 427, 44-50. doi: 10. 1016/j.virol.2012.02.004

Engelman, A. (1999). In vivo analysis of retroviral integrase structure and function. Adv. Virus Res. 52, 411-426. doi: 10.1016/S0065-3527(08)60309-7

Erwin, J. A., Marchetto, M. C., and Gage, F. H. (2014). Mobile DNA elements in the generation of diversity and complexity in the brain. Nat. Rev. Neurosci. 15, 497-506. doi: 10.1038/nrn3730

\section{FUNDING}

This work was supported by the Natural Sciences and Engineering Research Council of Canada (NSERC) through a Discovery grant for RD (RGPIN-2016-05761).

\section{ACKNOWLEDGMENT}

We would like to thank Mamneet Manghera and Alycia Magnusson for editorial suggestions.

Frank, O., Giehl, M., Zheng, C., Hehlmann, R., Leib-Mosch, C., and Seifarth, W. (2005). Human endogenous retrovirus expression profiles in samples from brains of patients with schizophrenia and bipolar disorders. J. Virol. 79, 10890 10901. doi: 10.1128/JVI.79.17.10890-10901.2005

Gasior, S. L., Wakeman, T. P., Xu, B., and Deininger, P. L. (2006). The human LINE-1 retrotransposon creates DNA double-strand breaks. J. Mol. Biol. 357, 1383-1393. doi: 10.1016/j.jmb.2006.01.089

Gerard, A., Soler, N., Segeral, E., Belshan, M., and Emiliani, S. (2013). Identification of low molecular weight nuclear complexes containing integrase during the early stages of HIV-1 infection. Retrovirology 10:13. doi: 10.1186/1742-469010-13

Golan, M., Hizi, A., Resau, J. H., Yaal-Hahoshen, N., Reichman, H., Keydar, I., et al. (2008). Human endogenous retrovirus (HERV-K) reverse transcriptase as a breast cancer prognostic marker. Neoplasia 10, 521-533. doi: 10.1593/neo. 07986

Gunthard, H. F., Saag, M. S., Benson, C. A., del Rio, C., Eron, J. J., Gallant, J. E., et al. (2016). Antiretroviral drugs for treatment and prevention of HIV infection in adults: 2016 recommendations of the international antiviral society-USA panel. JAMA 316, 191-210. doi: 10.1001/jama.2016.8900

Hanahan, D., and Weinberg, R. A. (2011). Hallmarks of cancer: the next generation. Cell 144, 646-674. doi: 10.1016/j.cell.2011.02.013

Hare, S., Gupta, S. S., Valkov, E., Engelman, A., and Cherepanov, P. (2010). Retroviral intasome assembly and inhibition of DNA strand transfer. Nature 464, 232-236. doi: 10.1038/nature08784

Hossain, M. A., Ali, M. K., and Shin, C. G. (2013). Structural and functional insights into foamy viral integrase. Viruses 5, 1850-1866. doi: 10.3390/v5071850

Jayappa, K. D., Ao, Z., and Yao, X. (2012). The HIV-1 passage from cytoplasm to nucleus: the process involving a complex exchange between the components of HIV-1 and cellular machinery to access nucleus and successful integration. Int. J. Biochem. Mol. Biol. 3, 70-85.

Johnson, B. C., Metifiot, M., Ferris, A., Pommier, Y., and Hughes, S. H. (2013). A homology model of HIV-1 integrase and analysis of mutations designed to test the model. J. Mol. Biol. 425, 2133-2146. doi: 10.1016/j.jmb.2013. 03.027

Jones, R. B., Song, H., Xu, Y., Garrison, K. E., Buzdin, A. A., Anwar, N., et al. (2013). LINE-1 retrotransposable element DNA accumulates in HIV-1-infected cells. J. Virol. 87, 13307-13320. doi: 10.1128/JVI.02257-13

Kanters, S., Vitoria, M., Doherty, M., Socias, M. E., Ford, N., Forrest, J. I., et al. (2016). Comparative efficacy and safety of first-line antiretroviral therapy for the treatment of HIV infection: a systematic review and network meta-analysis. Lancet HIV 3, e510-e520. doi: 10.1016/S2352-3018(16)30091-1

Kitamura, Y., Ayukawa, T., Ishikawa, T., Kanda, T., and Yoshiike, K. (1996). Human endogenous retrovirus K10 encodes a functional integrase. J. Virol. 70, 3302-3306.

Konsavage, W. M. Jr., Sudol, M., Lee, N. E., and Katzman, M. (2007). Retroviral integrases that are improved for processing but impaired for joining. Virus Res. 125, 198-210. doi: 10.1016/j.virusres.2007.01.006

Koyama, T., Sun, B., Tokunaga, K., Tatsumi, M., and Ishizaka, Y. (2013). DNA damage enhances integration of HIV-1 into macrophages by overcoming integrase inhibition. Retrovirology 10:21. doi: 10.1186/1742-4690-10-21

Kushima, I., Aleksic, B., Nakatochi, M., Shimamura, T., Shiino, T., Yoshimi, A., et al. (2016). High-resolution copy number variation analysis of schizophrenia in Japan. Mol. Psychiatry doi: 10.1038/mp.2016.88 [Epub ahead of print]. 
Kvaratskhelia, M., Sharma, A., Larue, R. C., Serrao, E., and Engelman, A. (2014). Molecular mechanisms of retroviral integration site selection. Nucleic Acids Res. 42, 10209-10225. doi: 10.1093/nar/gku769

Lee, Y. N., Malim, M. H., and Bieniasz, P. D. (2008). Hypermutation of an ancient human retrovirus by APOBEC3G. J. Virol. 82, 8762-8770. doi: 10.1128/JVI. 00751-08

Lesbats, P., Engelman, A. N., and Cherepanov, P. (2016). Retroviral DNA Integration. Chem. Rev. 116, 12730-12757. doi: 10.1021/acs.chemrev.6b0 0125

Letendre, S. L., Mills, A. M., Tashima, K. T., Thomas, D. A., Min, S. S., Chen, S., et al. (2014). ING116070: a study of the pharmacokinetics and antiviral activity of dolutegravir in cerebrospinal fluid in HIV-1-infected, antiretroviral therapynaive subjects. Clin. Infect. Dis. 59, 1032-1037. doi: 10.1093/cid/ciu477

Li, X., Koh, Y., and Engelman, A. (2012). Correlation of recombinant integrase activity and functional preintegration complex formation during acute infection by replication-defective integrase mutant human immunodeficiency virus. J. Virol. 86, 3861-3879. doi: 10.1128/JVI.06386-11

Li, X., Krishnan, L., Cherepanov, P., and Engelman, A. (2011). Structural biology of retroviral DNA integration. Virology 411, 194-205. doi: 10.1016/j.virol.2010. 12.008

Lord, C. J., and Ashworth, A. (2012). The DNA damage response and cancer therapy. Nature 481, 287-294. doi: 10.1038/nature10760

Maizels, N. (2015). G4-associated human diseases. EMBO Rep. 16, 910-922. doi: 10.15252/embr.201540607

Meeker, R. B., Asahchop, E., and Power, C. (2014). The brain and HAART: collaborative and combative connections. Curr. Opin. HIV AIDS 9, 579-584. doi: 10.1097/COH.0000000000000110

Mills, R. E., Bennett, E. A., Iskow, R. C., and Devine, S. E. (2007). Which transposable elements are active in the human genome? Trends Genet. 23, 183-191. doi: 10.1016/j.tig.2007.02.006

Mortelmans, K., Wang-Johanning, F., and Johanning, G. L. (2016). The role of human endogenous retroviruses in brain development and function. APMIS 124, 105-115. doi: 10.1111/apm.12495

Mousnier, A., Kubat, N., Massias-Simon, A., Segeral, E., Rain, J. C., Benarous, R., et al. (2007). von Hippel Lindau binding protein 1-mediated degradation of integrase affects HIV-1 gene expression at a postintegration step. Proc. Natl. Acad. Sci. U.S.A. 104, 13615-13620. doi: 10.1073/pnas.0705162104

Mullins, C. S., and Linnebacher, M. (2012). Human endogenous retroviruses and cancer: causality and therapeutic possibilities. World J. Gastroenterol. 18, 6027-6035. doi: 10.3748/wjg.v18.i42.6027

Nightingale, S., Winston, A., Letendre, S., Michael, B. D., McArthur, J. C., Khoo, S., et al. (2014). Controversies in HIV-associated neurocognitive disorders. Lancet Neurol. 13, 1139-1151. doi: 10.1016/S1474-4422(14)70137-1

O'Connor, M. J. (2015). Targeting the DNA damage response in cancer. Mol. Cell. 60, 547-560. doi: 10.1016/j.molcel.2015.10.040

Romanish, M. T., Cohen, C. J., and Mager, D. L. (2010). Potential mechanisms of endogenous retroviral-mediated genomic instability in human cancer. Semin. Cancer Biol. 20, 246-253. doi: 10.1016/j.semcancer.2010.05.005
Rubio, M. D., Wood, K., Haroutunian, V., and Meador-Woodruff, J. H. (2013). Dysfunction of the ubiquitin proteasome and ubiquitin-like systems in schizophrenia. Neuropsychopharmacology 38, 1910-1920. doi: 10.1038/npp. 2013.84

Ryan, E. L., Hollingworth, R., and Grand, R. J. (2016). Activation of the DNA damage response by RNA viruses. Biomolecules 6:2. doi: 10.3390/biom6010002

Seifarth, W., Frank, O., Zeilfelder, U., Spiess, B., Greenwood, A. D., Hehlmann, R., et al. (2005). Comprehensive analysis of human endogenous retrovirus transcriptional activity in human tissues with a retrovirus-specific microarray. J. Virol. 79, 341-352. doi: 10.1128/JVI.79.1.341-352.2005

Smith, C. L., Bolton, A., and Nguyen, G. (2010). Genomic and epigenomic instability, fragile sites, schizophrenia and autism. Curr. Genomics 11, 447-469. doi: 10.2174/138920210793176001

Stetson, D. B., Ko, J. S., Heidmann, T., and Medzhitov, R. (2008). Trex1 prevents cell-intrinsic initiation of autoimmunity. Cell 134, 587-598. doi: 10.1016/j.cell. 2008.06.032

Suzuki, Y., and Chew, M. L. (2012). Role of host-encoded proteins in restriction of retroviral integration. Front. Microbiol. 3:227. doi: 10.3389/fmicb.2012.00227

Van Maele, B., Busschots, K., Vandekerckhove, L., Christ, F., and Debyser, Z. (2006). Cellular co-factors of HIV-1 integration. Trends Biochem. Sci. 31, 98-105. doi: 10.1016/j.tibs.2005.12.002

Varatharajan, L., and Thomas, S. A. (2009). The transport of anti-HIV drugs across blood-CNS interfaces: summary of current knowledge and recommendations for further research. Antiviral Res. 82, A99-A109. doi: 10.1016/j.antiviral.2008. 12.013

Wiebe, M. S., and Jamin, A. (2016). The Barrier to autointegration factor: interlocking antiviral defense with genome maintenance. J. Virol. 90, 38063809. doi: 10.1128/JVI.00178-16

Wolkowicz, U. M., Morris, E. R., Robson, M., Trubitsyna, M., and Richardson, J. M. (2014). Structural basis of Mos1 transposase inhibition by the anti-retroviral drug Raltegravir. ACS Chem. Biol. 9, 743-751. doi: 10.1021/cb400791u

Yoder, K. E., and Bushman, F. D. (2000). Repair of gaps in retroviral DNA integration intermediates. J. Virol. 74, 11191-11200. doi: 10.1128/JVI.74.23. 11191-11200.2000

Zheng, R., Jenkins, T. M., and Craigie, R. (1996). Zinc folds the N-terminal domain of HIV-1 integrase, promotes multimerization, and enhances catalytic activity. Proc. Natl. Acad. Sci. U.S.A. 93, 13659-13664. doi: 10.1073/pnas.93.24.13659

Conflict of Interest Statement: The authors declare that the research was conducted in the absence of any commercial or financial relationships that could be construed as a potential conflict of interest.

Copyright (c) 2016 Bray, Turnbull, Hebert and Douville. This is an open-access article distributed under the terms of the Creative Commons Attribution License (CC BY). The use, distribution or reproduction in other forums is permitted, provided the original author(s) or licensor are credited and that the original publication in this journal is cited, in accordance with accepted academic practice. No use, distribution or reproduction is permitted which does not comply with these terms. 